\title{
Structural and functional analysis of the roles of the HCV 5' NCR miR122-dependent long-range association and SLVI in genome translation and replication
}

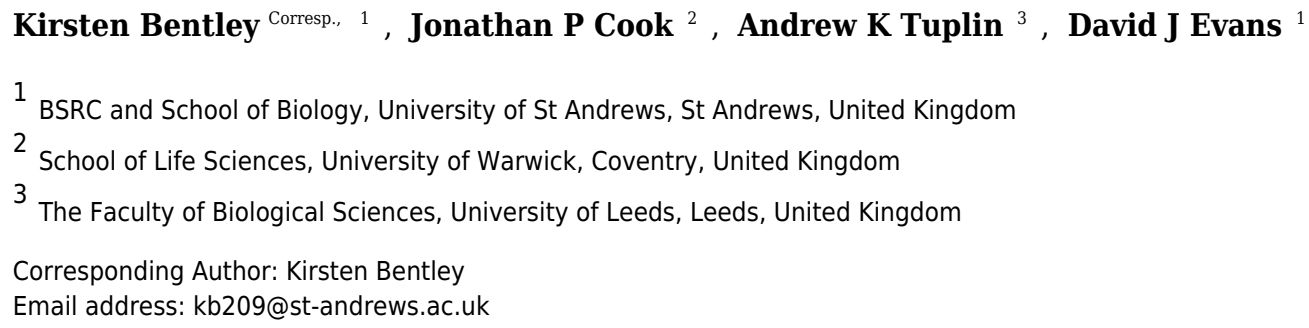

The hepatitis C virus RNA genome possesses a variety of conserved structural elements, in both coding and non-coding regions, that are important for viral replication. These elements are known or predicted to modulate key life cycle events, such as translation and genome replication, some involving conformational changes induced by long-range RNARNA interactions. One such element is SLVI, a stem-loop (SL) structure located towards the 5 ' end of the core protein-coding region. This element forms an alternative RNA-RNA interaction with complementary sequences in the 5'UTR that are independently involved in the binding of the cellular microRNA 122 (miR122). The switch between 'open' and 'closed' structures involving SLVI has previously been proposed to modulate translation, with lower translation efficiency associated with the 'closed' conformation. In the current study, we have used selective 2'-hydroxyl acylation analysed by primer extension (SHAPE) to validate this RNA-RNA interaction in the absence and presence of miR122. We show that the LRA only forms in the absence of miR122, or otherwise requires the blocking of miR122 binding combined with substantial disruption of SLVI. Using site-directed mutations introduced to promote open or closed conformations of the LRA we demonstrate no correlation between the conformation and the translation phenotype. In addition, we observed no influence on virus replication compared to unmodified genomes. The presence of SLVI is well-documented to suppress translation, but these studies demonstrate that this is not due to its contribution to the LRA. We conclude that, although there are roles for SLVI in translation, the LRA is not a riboswitch regulating the translation and replication phenotypes of the virus. 
1 Structural and functional analysis of the roles of the HCV 5' NCR miR122-dependent long-range

2 association and SLVI in genome translation and replication.

3

4

$5 \quad$ Kirsten Bentley ${ }^{1 *}$, Jonathan P. Cook ${ }^{2}$, Andrew K. Tuplin ${ }^{3}$ and David J. Evans ${ }^{1}$

6

7 Affiliations:

$8 \quad{ }^{1}$ BSRC and School of Biology, North Haugh, University of St Andrews, Fife, UK.

$9{ }^{2}$ School of Life Sciences, University of Warwick, Coventry, UK.

$10{ }^{3}$ The Faculty of Biological Sciences, University of Leeds, Leeds, UK.

11

$12{ }^{*}$ Communicating author: Kirsten Bentley, kb209@st-andrews.ac.uk.

13

14

15

16

17

18

19

20

21

22

23

24

25

26

27

28

29

30

31 
$\underline{\text { Abstract }}$

37

The hepatitis $\mathrm{C}$ virus RNA genome possesses a variety of conserved structural elements, in both coding and non-coding regions, that are important for viral replication. These elements are known or predicted to modulate key life cycle events, such as translation and genome

41

42 43

44 45 46 47 48 49 50 51

60

61 Hepatitis $\mathrm{C}$ virus (HCV) belongs to the genus Hepacivirus in the family Flaviviridae and, 62 despite the recent development of novel and effective therapies (Gao et al. 2010; Lawitz et al. replication, some involving conformational changes induced by long-range RNA-RNA interactions. One such element is SLVI, a stem-loop (SL) structure located towards the 5' end of the core protein-coding region. This element forms an alternative RNA-RNA interaction with complementary sequences in the 5'UTR that are independently involved in the binding of the cellular microRNA 122 (miR122). The switch between 'open' and 'closed' structures involving SLVI has previously been proposed to modulate translation, with lower translation efficiency associated with the 'closed' conformation. In the current study, we have used selective 2'hydroxyl acylation analysed by primer extension (SHAPE) to validate this RNA-RNA interaction in the absence and presence of miR122. We show that the LRA only forms in the absence of miR122, or otherwise requires the blocking of miR122 binding combined with substantial disruption of SLVI. Using site-directed mutations introduced to promote open or closed conformations of the LRA we demonstrate no correlation between the conformation and the translation phenotype. In addition, we observed no influence on virus replication compared to unmodified genomes. The presence of SLVI is well-documented to suppress translation, but these studies demonstrate that this is not due to its contribution to the LRA. We conclude that, although there are roles for SLVI in translation, the LRA is not a riboswitch regulating the translation and replication phenotypes of the virus.

\section{$\underline{\text { Introduction }}$}


63 2013; Welzel et al. 2017), infects approximately 185 million people globally, causing significant 64 levels of chronic liver disease and hepatocellular carcinoma (Mohd Hanafiah et al. 2013). Like 65 other flaviviruses HCV possesses a single-stranded, positive(mRNA)-sense genome packaged 66 into an enveloped virus particle (Chambers et al. 1990). The virus genome expresses a single 67 extensive open reading frame (ORF), flanked by highly structured 5' and 3' untranslated regions 68 (UTRs) which, upon delivery to the cytoplasm, is translated to yield a single polyprotein. The 69 latter is co- and post-translationally processed to generate the proteins required for genome 70 replication and particle formation. Thereafter, in a relatively poorly-understood process the 71 genome must act as the template for both translation of the polyprotein and replication resulting in new progeny genomes and, eventually, virus particles. The two processes of translation and replication, unless temporally separated or compartmentalised, must be mutually exclusive and are therefore likely to be controlled.

The limited coding capacity of small RNA viruses necessitates the genome being multi-

76

functional, with control of key events in the replication cycle being influenced by the presence of RNA secondary structures involved in either RNA-RNA interactions and/or the recruitment of viral or host factors (reviewed in (Li \& Nagy 2011; Nicholson \& White 2014; Tuplin 2015)). For $\mathrm{HCV}$, a number of RNA structures and RNA interactions have been identified throughout the genome and linked to a variety of functional roles (reviewed in (Adams et al. 2017; Niepmann et al. 2018)). Known or predicted conformational changes induced by long-range RNA-RNA interactions in the HCV genome have been described as molecular switches, potentially modulating translation by facilitating the switch from protein synthesis to genome replication (Romero-López et al. 2014; Shetty et al. 2013; Tuplin et al. 2012). The identification of the structures that form the core of these 'switches', and the dissection of the underlying molecular mechanism by which they work provides important insights into the replication of HCV and, by extrapolation, related viruses.

The HCV 5'UTR contains RNA signals essential for translation and replication and is an extensively structured region containing four stem-loop domains (SLI-IV; RNA structure naming conventions are detailed in Materials and Methods). Domains SLII-IV of the 5'UTR, along with the first 12-30 nucleotides of the core protein coding sequence, form the internal ribosome entry site (IRES) involved in cap-independent initiation of viral translation (Friebe et al. 2001; Reynolds et al. 1995). Two additional structures within the core coding region, SLV and SLVI, 
94 have also been implicated as important RNA elements in viral replication (McMullan et al.

95 2007). SLVI is a short stem-loop consisting of 54 paired bases, two sub-terminal bulge loops and

96 a terminal loop of 6 nucleotides (Tuplin et al. 2004). In addition to forming functional RNA

97 structures, the 5'UTR provides a platform for recruitment of the liver-specific microRNA 122

98 (miR122). In contrast to the typically repressive roles of cellular miRNAs, binding of miR122 to

99 two seed sites (S1 and S2) near the 5' end of the 5'UTR is critical for replication of HCV (Jangra

100 et al. 2010; Jopling et al. 2008; Jopling et al. 2005), as well as stabilising the genome and

101 providing protection against degradation (Li et al. 2013; Sedano \& Sarnow 2014; Shimakami et

102 al. 2012).

103 Intriguingly, a sequence spanning the miR122 seed sites has also been demonstrated to

104 anneal to complementary sequences that form the basal stem of SLVI. Mutation analysis of

105 sequences in these regions had previously suggested a role for such an interaction in controlling

106 translation (Honda et al. 1999; Kim et al. 2003). Subsequently, several methodologies, including

107 RNase cleavage assays and atomic force microscopy, have been used to probe this region and

108 map conformational changes in RNA structure involving these sequences (Beguiristain et al.

109 2005; Díaz-Toledano et al. 2009; García-Sacristán et al. 2015). Consequently, as identified by

110 Díaz-Toledano (Díaz-Toledano et al. 2009), a conformational change is induced via the 3-way

111 interplay of miR122, the 5'UTR and SLVI and this may function as a molecular switch,

112 regulating translation and replication. In the simplest scenario, two mutually-exclusive, higher-

113 order RNA structures are predicted as possible; 'open', in which miR122 is bound to the 5'UTR,

114 and the 'closed' structure, in which there is a long-range association (LRA), between the

115 miR122-binding site in the 5'UTR and the base of SLVI in the core-coding region (Fig. 1A).

116 In this study we have utilised a panel of defined 5'UTR and SLVI mutations to

117 investigate a translation-modulating role for the switch from the 'closed' to the 'open'

118 conformation of this potential molecular switch. SHAPE (selective 2'-hydroxyl acylation

119 analysed by primer extension; (Merino et al. 2005)) analysis confirms previous reports that the

120 change from the 'closed' to the 'open' conformation is influenced by the availability of miR122

121 (Díaz-Toledano et al. 2009). We further show that the 'closed' conformation is only achieved in

122 the absence of miR122, or when both miR122 binding is blocked and base pairing within the

123 basal stem of SLVI is prevented. Importantly, we were unable to correlate the 'open' or 'closed'

124 conformations with specific translation phenotypes in either the presence or absence of miR122, 
125 and replication of the virus genome was apparently unaffected by these gross structural changes.

126 Therefore, whilst the presence of SLVI is undoubtedly important for regulation of translation, the

127 LRA between the miR122 binding sites in the 5'UTR and the base of SLVI, necessary for the

128 formation of the 'closed' conformation, may not have an important regulatory role in either HCV

129 replication or translation.

130

131 Materials and Methods

132

133 Cell culture and transfection

$134 \quad$ Huh 7.5 human hepatocellular carcinoma cells were maintained at $37^{\circ} \mathrm{C}, 5 \% \mathrm{CO}_{2}$, in

135 Dulbecco's modified minimal essential medium (DMEM) (Sigma Aldrich) supplemented with

$13610 \%(\mathrm{v} / \mathrm{v})$ heat-inactivated foetal bovine serum (FBS), 1\% non-essential amino acids and 2

137 mM L-glutamine (GIBCO, Life Technologies; DMEM-FBS). HeLa cells were maintained at

$13837^{\circ} \mathrm{C}, 5 \% \mathrm{CO}_{2}$, in DMEM supplemented with 10\% (v/v) FBS. Cells were seeded in 24-well

139 plates at $1 \times 10^{5}$ cells/well for translation assays, or $0.8 \times 10^{5}$ cells/well for replicon assays, in

140 DMEM-FBS $18 \mathrm{~h}$ prior to transfection.

141 Transfections were carried out with $500 \mathrm{ng}$ RNA and $2 \mu$ l of Lipofectamine 2000 (Life

142 Technologies) as per manufacturers' instructions. In addition, for translation assays $5 \mathrm{ng}$ of a

143 capped and polyadenylated Renilla luciferase RNA was added as a transfection control.

144 Transfection media was replaced at $4 \mathrm{~h}$ with fresh DMEM-FBS. Cell lysates were harvested for

145 analysis at $4 \mathrm{~h}$ (translation assay) or 4, 21, 28, and $45 \mathrm{~h}$ (replicon assay). Briefly, cells were

146 washed twice with PBS and lysed with $0.1 \mathrm{ml} /$ well Glo Lysis buffer (Promega) for 15 min with

147 shaking. Luciferase readings were determined with Dual-Luciferase (translation assays), or

148 Luciferase (replicon assays), Assay System Kits (Promega) as per manufacturers' instruction.

149

150 HCV cDNA plasmids, JFH-1 replicon construction and mutagenesis

151 A variety of numbering or naming schemes have been used to specify RNA stem-loop

152 structures in the HCV genome. Although the most logically extendable scheme involves

153 numbering structures according to the location of the 5 ' nucleotide within the relevant genome

154 region in an $\mathrm{H} 77$ reference sequence (Kuiken et al. 2006), for consistency with previous work on

155 the HCV IRES and long-range structures between coding and non-coding regions, we use the 
156 SLI-VI scheme here. For comparison, SLVI is SL87 according to the Kuiken et al.

157 nomenclature, due to the 5 most nucleotide being located at $\mathrm{nt} 87$ of the core coding region.

158

A JFH-1 based translation-only reporter construct containing an extended core sequence

159 (CE), to include SLV and VI - designated pJFH1-CEtrans - was generated via modification of

160 pJFH1-luc-trans: $\triangle$ NS5B described previously (Tuplin et al. 2015). Overlap PCR was used to

161 generate a DNA consisting of the 5' end of JFH-1, including the first 174 nt of JFH-1 core, fused

162 to the 5 ' end of Firefly luciferase. An $\mathrm{Agel} / \mathrm{Xba \textrm {I }}$ fragment was ligated into similarly digested

163 pJFH1-luc-trans: $\triangle \mathrm{NS} 5 \mathrm{~B}$ to generate $\mathrm{pJFH1-CEtrans.}$

164

Mutations at miR122 seed site 1 (S1) or/and SLVI were introduced into pJFH1-CEtrans

165 using the QuikChange II site-directed mutagenesis kit as per manufacturers' instructions

166 (Agilent). To disrupt miR122 binding, point mutations $\mathrm{U}_{25} \mathrm{~A}$ and $\mathrm{G}_{28} \mathrm{C}$ were introduced into the

167 miR122 S1 site to generate pJFH1-CEtrans-S1 (F:5'-GGCGACACㅁCCCCCATGAATCACTC-

168 3' and R:5'-GAGTGATTCATGGGGGTGTGTCGCC-3'). To disrupt the SLVI structure point

169 mutations $\mathrm{C}_{436} \mathrm{G}$ and $\mathrm{A}_{439} \mathrm{U}$ (F:5'-CCAGATCGTTGGGGGTATACTTGTTGC-3' and R:5'-

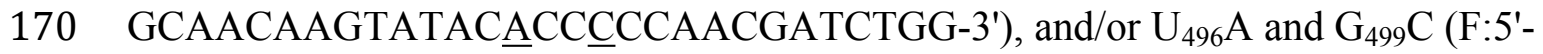

171 CGACAAGGAAAACÁTCEGGAGCGGTCCCAGC-3' and R:5'-

172 GCTGGGACCGCTCGGATGTTTTCCTTGTCG-3'), were introduced into the 5' and/or 3' stem

173 of SLVI to generate $\mathrm{pJFH1}$-CEtrans-L, pJFH1-CEtrans-R and pJFH1-CEtrans-L/R respectively.

174 Mutants pJFH1-CEtrans-S1/L, pJFH1-CEtrans-S1/R and pJFH1-CEtrans-S1/L/R, containing

175 both the miR122 S1 and SLVI mutations were generated by site-directed mutagenesis of pJFH1-

176 CEtrans-L, pJFH1-CEtrans-R and pJFH1-CEtrans-L/R with the pJFH1-CEtrans-S1 primers

177 described above. All mutations were confirmed by sequence analysis.

178 A JFH-1 replicon, containing the extended core sequence as above, and designated

179 pJFH1-CErep, was designed based on Conlb-luc-rep (Tuplin et al. 2015). Overlap PCR was

180 used to replace the Con $1 \mathrm{~b} 5$ 'UTR with that of JFH-1 within Con1b-rep-luc to generate pJFH1-

181 5'UTR-Conlb-rep. A second overlap PCR generated a DNA containing the 3' end of Firefly

182 luciferase, the EMCV IRES and an ATG codon for NS3, and introduced into the previously

183 described pJ6/JFH-1 (Lindenbach et al. 2005) to generate pJFH1-EMCV. An Sbfl/NotI fragment

184 from pJFH1-5'UTR-Con1b-rep containing the JFH-1 5'UTR, poliovirus IRES and Firefly

185 luciferase, was inserted into similarly digested pJFH1-EMCV to generate pJFH1-rep. An

$186 \mathrm{Sbfl} / \mathrm{PmeI}$ fragment of $\mathrm{pJFH}-1-\mathrm{CEtrans}$ was inserted into similarly digested pJFH1-rep to 
187 generate the core-extended JFH-1 replicon, pJFH1-CErep. Mutations at miR122 S1 and/or SLVI

188 were introduced through Sbfl/PmeI digestion of the appropriate pJFH1-CEtrans plasmid, and

189 ligation into pJFH1-CErep. This resulted in replicon constructs pJFH1-CErep-S1, pJFH1-CErep-

190 L, pJFH1-CErep-R, pJFH1-CErep-L/R, pJFH1-CErep-S1/L, pJFH1-CErep-S1/R and pJFH1-

191 CErep-S1/L/R.

192

193 miR122 duplexing and electrophoretic mobility shift assay

194 Native (miR122), complementary (miR122-Comp) and S1 mutant (S1-miR122) miR122

195 RNAs (5'-UGGaGUGUGACAAUGGUGUUUGU-3', 5'-

196 AAACGCCAUUAUCACACUAAAUA-3' and 5'-GGGUGUGUGACAAUGGUGUUUGU-3')

197 were synthesized by Integrated DNA Technologies along with an RNA oligonucleotide

198 corresponding to nucleotides 1-50 of the JFH-1 strain of HCV (JFH1 $\left.{ }^{1-50}\right)$.

199 For addition of miR122 to replicon assays, miR122 RNA (10 mM) was duplexed with 200 miR122-Comp (10 mM) in a final concentration of $100 \mathrm{mM} \mathrm{HEPES,} 5 \mathrm{mM} \mathrm{MgCl}_{2}$, heated at $20165^{\circ} \mathrm{C}$ for $5 \mathrm{~min}$ and cooled slowly to room temperature.

202 For electrophoretic mobility shift assays (EMSA) JFH1 ${ }^{1-50}$ RNA (10 pmol) was heated 203 for $5 \mathrm{~min}$ at $65^{\circ} \mathrm{C}$ followed by cooling to $35^{\circ} \mathrm{C}$ for $1 \mathrm{~min}$ in a $5 \mu 1$ volume containing $100 \mathrm{mM}$ 204 HEPES ( $\mathrm{pH}$ 7.6), $100 \mathrm{mM} \mathrm{KCl}$ and $5 \mathrm{mM} \mathrm{MgCl}_{2}$. miR122 RNAs were added at molar ratios of $2050,0.5,1,1.5,2,3$ and 5 and incubated for a further $30 \mathrm{~min}$ at $37^{\circ} \mathrm{C}$. An equal volume of loading 206 dye ( $30 \%$ glycerol, $0.5 \times \mathrm{TBE}$ and $5 \mathrm{mM} \mathrm{MgCl}_{2}$ ) was added and RNA complexes separated by 207 non-denaturing gel electrophoresis (15\% 29:1 acrylamide:bisacrylamide, $0.5 \times \mathrm{TBE}$, and $5 \mathrm{mM}$ $208 \mathrm{MgCl}_{2}$ ) at $150 \mathrm{~V}, 4^{\circ} \mathrm{C}, 3 \mathrm{~h}$ using a BioRad MiniProtean III gel system. Gels were stained with 209 SYBR Gold (Life Technologies) and RNA visualized using a Typhoon FLA 9500 (GE

210 Healthcare).

211

\section{In vitro RNA transcription}

213 RNA transcripts were synthesized using a HiScribe ${ }^{\mathrm{TM}}$ T7 High Yield RNA Synthesis Kit

214 (NEB), as per manufacturers' instructions, with $1 \mu \mathrm{g}$ of DNA template linearized with $B s p \mathrm{HI}$ 215 (for translation templates) or terminated with a 3' cis-acting ribozyme from an $M l u \mathrm{I}$ linearized 216 template (for replicon templates). Newly transcribed RNA was column-purified using a GeneJET 217 RNA Purification Kit (ThermoFisher Scientific). 
RNA modification for SHAPE

220

$10 \mathrm{pmol}$ of translation construct-derived RNA transcripts were prepared in $10 \mu \mathrm{l}$ of $0.5 \times$

221 Tris-EDTA ( $\mathrm{pH} \mathrm{8.0)} \mathrm{(TE),} \mathrm{denatured} \mathrm{at} 95^{\circ} \mathrm{C}$ for $3 \mathrm{~min}$ and incubated on ice for $3 \mathrm{~min}$ prior to

222 addition of $6 \mu \mathrm{l}$ of either a $5 \mathrm{mM}$ or $10 \mathrm{mM} \mathrm{MgCl}_{2}$ folding buffer [333 mM HEPES (pH8.0), 333

$223 \mathrm{mM} \mathrm{NaCl}$ and, $16.5 \mathrm{mM}$ or $33 \mathrm{mM} \mathrm{MgCl}_{2}$ ]. Samples were allowed to refold at $37^{\circ} \mathrm{C}$ for $20 \mathrm{~min}$

224 before being divided in half and incubated with either $1 \mu \mathrm{l}$ of $100 \mathrm{mM}$ N-methylisatoic

225 anhydride (NMIA) dissolved in DMSO, or $1 \mu 1$ of DMSO, at $37^{\circ} \mathrm{C}$ for $45 \mathrm{~min}$. For reactions in

226 the presence of miR122 a 3 molar excess of miR122 was added prior to addition of folding

227 buffer. Modified RNAs were column-purified using a GeneJET RNA Purification Kit

228 (ThermoFisher Scientific) to remove miR122 prior to reverse transcription.

229

230

231

\section{$5^{\prime}-\left[{ }^{32} \mathbf{P}\right]-p r i m e r$ labelling}

A total of $60 \mu \mathrm{M}$ of primer was incubated with 10 units of T4 polynucleotide kinase

232 (NEB), $2 \mu \mathrm{l}$ of supplied $10 \times$ buffer and $10 \mu \mathrm{\gamma}-\left[{ }^{32} \mathrm{P}\right]-\mathrm{ATP}\left(3.7 \times 10^{6} \mathrm{~Bq}\right.$; Perkin Elmer $)$ at $37^{\circ} \mathrm{C}$

233 for $30 \mathrm{~min}$ followed by heat inactivation at $65^{\circ} \mathrm{C}$ for $20 \mathrm{~min}$. Radiolabelled primers were purified 234 by separation on Sephadex G-25 Quick Spin Oligo Columns (Roche).

\section{Primer extension for SHAPE}

NMIA- or DMSO-treated RNA in $0.5 \times \mathrm{TE}(10 \mu \mathrm{l})$ was mixed with $3 \mu \mathrm{l}$ of radiolabelled

238 primer, denatured at $95^{\circ} \mathrm{C}$ for $5 \mathrm{~min}$, annealed at $35^{\circ} \mathrm{C}$ for $5 \mathrm{~min}$ and chilled on ice for $2 \mathrm{~min}$.

239 Reverse transcription (RT) mix $(6 \mu \mathrm{l})$ was added $(5 \times$ First Strand Buffer, $5 \mathrm{mM}$ DTT, $0.5 \mathrm{mM}$

240 dNTPs; Life Technologies) and samples incubated at $55^{\circ} \mathrm{C}$ for 1 min prior to addition of $1 \mu l$ of

241 SuperScript ${ }^{\circledR} I I I$ (Life Technologies) and further incubation at $55^{\circ} \mathrm{C}$ for $30 \mathrm{~min}$. The RNA

242 template was degraded by addition of $1 \mu \mathrm{l}$ of $4 \mathrm{M} \mathrm{NaOH}$ and incubation at $95^{\circ} \mathrm{C}$ for 5 min before

243 addition of $29 \mu \mathrm{l}$ of acid stop mix (140 mM un-buffered Tris-HCl, 73\% formamide, $0.43 \times \mathrm{TBE}$,

24443 mM EDTA [pH 8.0], bromophenol blue and xylene cyanol dyes) and further incubation at

$24595^{\circ} \mathrm{C}$ for $5 \mathrm{~min}$. Dideoxynucleotide (ddNTP) sequencing markers were generated by the

246 extension of unmodified RNA with addition of $2 \mu \mathrm{l}$ of $20 \mathrm{mM}$ ddNTP (TriLink

247 BioTechnologies) prior to addition of RT mix. The cDNA extension products were separated by 
248 denaturing electrophoresis [7\% (19:1) acrylamide:bisacrylamide, $1 \times \mathrm{TBE}, 7 \mathrm{M}$ urea] at $70 \mathrm{~W}$ for

249 3-5 $\mathrm{h}$ depending on product sizes to be analysed. Gels were visualised with a phosphorimager

250 (Typhoon FLA 9500) and densitometry analysis carried out with ImageQuant TL 8.1 software

251 (GE Healthcare Life Sciences). Normalised reactivities indicating exposure of nucleotides in

252 predicted RNA structures were calculated as described previously (Tuplin et al. 2012).

253

$254 \underline{\text { Results }}$

255

256 Mutagenesis of a miR122 binding site and the sequences implicated in the LRA

257 The previously probed 'open' and 'closed' structural conformations are determined by 258 complementarity between the 5 ' nucleotides forming the miR122 binding site and the base of

259 SLVI in the core protein-coding region, together with the presence of exogenous miR122

260 (Beguiristain et al. 2005; Díaz-Toledano et al. 2009). The latter, by binding to the 5'UTR

261 sequences, inhibits the LRA and 'opens' the structure (Fig. 1A). This transition from a 'closed'

262 to an 'open' structure can be predicted bioinformatically using mfold (Zuker 2003) and bifold

263 RNA secondary structure prediction software (Reuter \& Mathews 2010), to demonstrate that if

264 the S1 site is occupied by miR122 the 'open' conformation with bound miR122 is energetically

265 more favourable (Fig. S1). To investigate the existence and potential functions of the alternative

266 conformations we first modified our existing JFH-1 translation reporter vector, JFH1-luc-

267 trans: $\triangle$ NS5B (Fig. 1B), to generate a core-extended (CE) version, JFH1-CEtrans, which

268 encompasses the first 174 nucleotides of the core-coding region, thus incorporating SLVI. A

269 JFH-1 based sub-genomic replicon, designated JFH1-CErep, was additionally constructed to

270 include the same core-extended sequence (Fig. 1B). For both JFH1-CEtrans and JFH1-CErep we

271 subsequently undertook a systematic mutagenesis of either, or both, of the complementary

272 sequences required for formation of the LRA.

273 First, using mfold structure prediction (Zuker 2003), we identified two sites within SLVI

274 at which synonymous substitutions could be introduced that should disrupt formation of the

275 structure (Fig. 1A). Substitutions $\mathrm{C}_{436} \mathrm{G}$ and $\mathrm{A}_{439} \mathrm{U}$ in the 5 ' stem of SLVI (designated ' $\mathrm{L}$ '

276 mutants) and $\mathrm{U}_{496} \mathrm{~A}$ and $\mathrm{G}_{499} \mathrm{C}$ in the 3' stem of SLVI (designated ' $\mathrm{R}$ ' mutants) independently

277 prevented base pairing of the basal stem of SLVI. Both $\mathrm{C}_{436}$ and $\mathrm{A}_{439}$ are implicated in the

278 formation of the 'closed' structure and consequently, 'L' mutants were predicted to additionally 
279 prevent the LRA due to disruption of the complementarity with the miR122 seed site 1 (S1).

280 Conversely, ' $R$ ' mutants would free the 5' sequences forming the basal stem of SLVI to

281 contribute solely to formation of the 'closed' structure. However, since formation of the 'closed'

282 structure would also be dependent on the S1 site being unoccupied by miR122, we also

283 introduced substitutions into the latter (at positions $\mathrm{U}_{25} \mathrm{~A}$ and $\mathrm{G}_{28} \mathrm{C}$ ) that were predicted to

284 prevent miR122 binding and at the same time would restore complementarity with the ' $\mathrm{L}$ '

285 mutations in SLVI (Fig. 1C, Table 1).

286 To verify that binding of miR122 to S1 was abrogated in the S1 mutants we conducted

287 electrophoretic mobility shift assays (EMSAs) using synthetic miR122 and an RNA

288 oligonucleotide corresponding to the first 50 nucleotides of JFH-1 (JFH1 $\left.{ }^{1-50}\right)$. With the addition

289 of miR122 to unmodified JFH1 ${ }^{1-50}$ we observed the expected two complexes with reduced

290 mobility, representative of binding of miR122 to both S1 and S2 seed sites. Saturation of both

291 seed sites was achieved upon addition of a 3:1 molar ratio of miR122:JFH1 ${ }^{1-50}$ (Fig. 2A), while

292 addition of an antisense miR122 RNA (miR122-Comp) showed no change in mobility (Fig. 2B).

293 In contrast to unmodified JFH1 ${ }^{1-50}, \mathrm{~S} 1$-mutated JFH1 $1^{1-50}$ only formed the faster migrating single

294 complex, even at a 5:1 molar ratio, indicating that miR122 remained bound to S2 alone (Fig.

295 2C). Restoration of both mobility-shifted complexes was achieved upon addition of a 50-50 mix

296 of unmodified and S1-modified miR122 (S1-miR122), the latter containing mutations

297 complementary to those introduced in S1 mutated JFH1 ${ }^{1-50}$ (Fig. 2D). These studies confirmed

298 that substitutions introduced to the $\mathrm{S} 1$ site were sufficient to disrupt miR122 binding to the $\mathrm{S} 1$

299 seed site, but that binding to the S2 seed site was unaffected, in agreement with similar mutation 300 analysis of miR122 binding (Mortimer \& Doudna 2013).

301 To investigate the influence on the conformation of the 5 ' end of the HCV RNA the L, R 302 and S1 mutations predicted to influence the 'open' or 'closed' conformation were introduced 303 individually, or in combination, into the core-extended translation and replicon reporters, JFH1304 CEtrans and JFH1-CErep respectively, and individual templates validated by sequence analysis 305 (Table 1).

306 
The LRA is detected in the absence, but not presence, of miR122

We have previously used SHAPE mapping to demonstrate a long-range interaction between the 3'UTR of the HCV genome and distal sequences located within the polyproteincoding region (Tuplin et al. 2012). These interactions occurred only in cis and were acutely sensitive to point mutations within the complementary regions. We were therefore confident SHAPE analysis could provide useful insights into the study of the LRA. Three regions of the HCV RNA were analysed to provide data on RNA structure: (1) the 5' base stem of SLVI, (2) the 3' base stem of SLVI and, (3) nts 1-80 of the 5'UTR. Unfortunately, the presence of a highly stable stem-loop (SLI; Fig. 1A) immediately 5 ' to the S1 miR122 binding site acted as a strong terminator during cDNA synthesis. Consequently, as others have previously found (Pang et al. 2012), the 5' end of the S1 miR122 binding site (nts 1-20) proved difficult to accurately map due to excessive background signal. Scrutiny of the predicted pattern of base pairing between the miR122 binding site and miR122 also shows that it is highly similar to that between the miR122 binding site and the $5^{\prime}$ base stem of SLVI. Together, these issues meant that the S1 region was not informative for defining the 'open' or 'closed' conformation. Determination of the 'closed' structure was therefore based primarily on the structure of SLVI. In particular, nucleotides 434435 (GG), which are predicted to be paired when involved in the LRA but unpaired in formation of the basal stem of SLVI, and the overall paired/unpaired nature of the 3' side of the basal stem of SLVI (nucleotides 494-507), which would be predominantly unpaired upon formation of the 'closed' structure (Fig. 1A, S1). Preliminary experiments showed that SHAPE mapping of the 5' regions of a variety of unmodified templates e.g. JFH1-CEtrans, JFH1-CErep, or a full-length in vitro transcribed RNA, resulted in the same NMIA reactivity and resulting structural predictions (data not shown). We conclude from this that the LRA interactions are essentially local in nature and are unaffected by distal sequences in the virus genome. All subsequent SHAPE mapping was conducted using JFH1-CEtrans as template.

We first compared the structural conformations of parental JFH1-CEtrans in the absence of miR122 during the RNA folding reaction, or with a 3:1 molar excess of miR122 to saturate binding to S1 and S2, as determined from EMSAs (Fig. 2A). In the presence of miR122 the basal stem of SLVI was predominantly NMIA-unreactive, indicating that the pairing through this region was in agreement with the structure predicted bioinformatically (Fig. 3A). Indeed, the 
338 reactivity of nucleotides 427-447 and 487-507 corresponded very well with both the predicted 339 and RNAse-probed structure of this region of SLVI (Tuplin et al. 2004). As additional validation 340 we determined the NMIA-reactivity of SLVI sequences in JFH1-CEtrans in the presence of a 341 locked-nucleic-acid (LNA) probe, J22. LNA J22 binds with high affinity to nts 21-37 of JFH-1

342 across the miR122 binding sites, allowing for the determination of the SLVI structure 343 independently of the reversible action of miR122 (Fig. 3C). The resulting SHAPE analysis 344 recapitulated the results observed in the presence of miR122, with little or no reactivity of 345 sequences known to form the basal stem of SLVI. These results support the previously probed 346 structure of SLVI indicating that, in the presence of miR122, the 'open' conformation 347 predominates.

348 miR122 is present at high levels in hepatic cells in which HCV replicates (Lagos-

349 Quintana et al. 2002). Nevertheless, since there might be compartmentalisation - in replication 350 complexes for example - where miR122 is limited or absent, we went on to investigate the 351 potential formation of the 'closed' structure by SHAPE in the absence of miR122 (Fig. 3B). 352 Under these conditions we observed gross changes to the structure of the basal region of SLVI. 353 The $\mathrm{G}_{434} \mathrm{G}_{435}$ motif - predicted to be a key interaction with the $\mathrm{S} 1$ site - are highly unreactive, 354 indicating that they are base paired. At the same time, the reactivity of the $3^{\prime}$ sequences of the 355 basal stem of SLVI increases. There are significant increases in exposure of nt 490-503 356 indicative of a more extensive opening out of the SLVI structure. We interpret this as the 357 formation of the 'closed' structure in the absence of miR122, despite the inability to measure the 358 reactivity of nucleotides within the S1 site. In contrast to the results of García-Sacristán et al. 359 (García-Sacristán et al. 2015), but perhaps indicative of differences between in vitro techniques, 360 we were unable to demonstrate a magnesium-dependent preference for the formation of the 361 'closed' structure while in the presence of miR122. We investigated the structure of the basal 362 stem of SLVI in the parental templates at an increased concentration of $10 \mathrm{mM} \mathrm{MgCl}_{2}$ and 363 determined that the 'open' conformation predominated, irrespective of the magnesium 364 concentration (Fig. S2).

365 Together, these results are in agreement with a previous conclusion by Díaz-Toledano et 366 al. obtained via RNase III cleavage assays (Díaz-Toledano et al. 2009), and are highly indicative 367 of an inhibitory role for miR122 in formation of the 'closed' structure. 
369

370

371

372

373

374

375

376

377

378

379

380

381

382

383

384

385

386

387

388

389

390

391

392

393

394

395

396

397

398

399

In the presence of miR122, the LRA is favoured only when both miR122 binding and the SLVI structure are disrupted

Having investigated the pairing of the basal stem of SLVI and the occurrence of the LRA in unmodified templates, we went on to study the influence of mutations introduced to prevent these interactions, or that we had previously shown prevent miR122 binding. All subsequent analyses were carried out in the presence of miR122.

We first analysed those templates predicted to preferentially form the 'open' conformation (Fig. 4). Modification of the S1 site in template JFH1-CEtrans-S1 (Fig. 4A), shown to abrogate miR122 binding (Fig. 2C), resulted in a NMIA-reactivity pattern almost indistinguishable from the unmodified parental template (compare Fig. 3A with Fig. 4A). Since the $\mathrm{U}_{25} \mathrm{~A}$ and $\mathrm{G}_{28} \mathrm{C}$ changes in the $\mathrm{S} 1$ mutant also prevents interaction with SLVI nts $\mathrm{A}_{439}$ and $\mathrm{C}_{436}$ respectively, this provides further support that this pattern of NMIA reactivity represents the 'open' conformation. Three additional modified templates, JFH1-CEtrans-L, JFH1-CEtrans-L/R and JFH1-CEtrans-S1/R, were also predicted to block the LRA by reducing the complementarity between nucleotides in the S1 site and the basal stem of SLVI (Fig. 1C). NMIA-reactivity of these three templates verified the predicted inhibition of the LRA, as evidenced by the high reactivity of the $\mathrm{G}_{434} \mathrm{G}_{435}$ motif (Fig. $4 \mathrm{~B}, \mathrm{C}, \mathrm{D}$ ). Interestingly, in comparison to the parental JFH1-CEtrans, all three templates displayed increases in reactivity in other regions, such as nts 427-433 (the 5' basal stem) or nts 491-495 (3' basal stem), suggesting that, while preventing the LRA, the introduced mutations may also lead to generation of an altered SLVI structure (compare line graph to black bars in Fig. 4B, C, D). The mfold predictions for the structure of SLVI containing mutations $\mathrm{C}_{436} \mathrm{G}$ and $\mathrm{A}_{439} \mathrm{U}$, and $\mathrm{U}_{496} \mathrm{~A}$ and $\mathrm{G}_{499} \mathrm{C}$, did not suggest formation of such an altered structure (data not shown) and it is not possible to deduce the precise structure from the NMIA-reactivity plots.

We next investigated the conformation of templates containing combinations of mutations that were predicted to favour the LRA and the 'closed' conformation: JFH1-CEtransR, JFH1-CEtrans-S1/L and JFH1-CEtrans-S1/L/R (Fig. 5). Unexpectedly, both JFH1-CEtrans-R and JFH1-CEtrans-S1/L/R failed to demonstrate the LRA, again as evidenced by the reactivity of the $\mathrm{G}_{434} \mathrm{G}_{435}$ motif, as well as the overall lack of reactivity in the 3' basal stem of SLVI that would be expected (Fig. 5A, B). As with JFH1-CEtrans-L and JFH1-CEtrans-L/R, we observed 
400 an overall increase in reactivity of the 5 ' basal stem nucleotides suggestive of a similar disruption

401 to the SLVI structure that was not predicted in mfold calculations (compare Fig. 5A and B with

402 Fig. 4B and C). However, these result suggest that despite significant disruption to the known

403 SLVI structure, the 'closed' structure is not the favoured conformation for the RNA template.

404 In contrast to all other modified templates JFH1-CEtrans-S1/L generated a NMIA-

405 reactivity plot matching that of parental JFH1-CEtrans in the absence of miR122, and is highly

406 indicative of the formation of the 'closed' structure (Fig. 5C). In comparison to a template in

407 which the 'closed' structure is blocked, i.e. parental JFH1-CEtrans in the presence of miR122,

408 the mean NMIA-reactivities of the JFH1-CEtrans-S1/ $\mathrm{L} \mathrm{G}_{434} \mathrm{G}_{435}$ motif were reduced from 0.38

409 and 1.16 to 0.26 and 0.22 respectively, highlighting a substantial change in the base paired state,

410 especially of $\mathrm{G}_{435}$. Similarly, the average reactivity of nts 490-507 in the 3' basal stem was

411 increased from 0.28 to 0.64 demonstrating the overall increase in reactivity expected when the $5^{\prime}$

412 basal stem of SLVI is bound to the miR122 S1 site in the 'closed' conformation.

413 Taken together the SHAPE analyses show that, in the presence of miR122, the 'closed"

414 conformation only exists when both miR122 binding at S1 is blocked, and nucleotide

415 complementarity between S1 and the 5' basal stem of SLVI is restored.

416

417 Phenotypic Characterisation of LRA-modified templates

418 Using SHAPE analyses we determined that, in the presence of miR122, the LRA

419 resulting in the 'closed' conformation, is highly unlikely to form. However, if HCV translation

420 and/or replication occur in locations or complexes in which miR122 is absent then the 'closed'

421 structure is the energetically favourable conformation (Fig. 3B), and as such may influence virus

422 translation and replication. We therefore used selected modified templates with demonstrated

423 changes in conformation, to investigate the effects of the LRA on translation and replication.

424 HCV IRES-mediated translation is known to require the first 12-30 nt of the core protein-

425 coding region (Reynolds et al. 1995). However, to study the effects on translation of the LRA

426 required the additional SLV-SLVI sequences included in the core-extended translation reporter

427 described above and utilised in SHAPE analysis (Fig.1B). We initially compared translation

428 from JFH1-luc-trans: $\triangle \mathrm{NS} 5 \mathrm{~B}$, containing the minimal core sequence, to the core-extended JFH1-

429 CEtrans reporter. In agreement with previous observations (Kim et al. 2003), translation was

430 significantly decreased ( 2.5-fold) with the inclusion of the extended core sequence (Fig. 6A). 
431 This reduction in translation is proposed to be a result of formation of the LRA (Kim et al. 2003)

432 and that a high proportion of the JFH1-CEtrans RNA templates exist in the 'closed'

433 conformation (Fig. 1A), and hence are unavailable for use by the cellular translation machinery.

434 As we have demonstrated that the LRA occurs only in the absence of miR122 (Fig. 3), this result

435 implies either the exclusion of miR122 from sites of translation or, an alternative role for the

436 sequences encompassing SLV and SLVI domains in regulating translation. If the observed

437 reduction is a result of the LRA, translation levels should be restored by mutations designed to

438 disrupt the LRA, so forcing the 'open' conformation, and repressed again by compensatory

439 substitutions that - although different from the parental template - restore the LRA and the

440 'closed' conformation.

441 We therefore compared translation from JFH1-CEtrans with selected modified templates

442 that had shown a distinct conformation in SHAPE analysis. We selected JFH1-CEtrans-S1 as a

443 representative of the 'open' conformation due to the SHAPE analysis most closely matching the

444 SLVI structure observed for parental JFH1-CEtrans. For the 'closed' conformation we were

445 limited to the study of JFH1-CEtrans-S1/L, as the only modified template in which the LRA was

446 demonstrated. In addition, we investigated JFH1-CEtrans-L/R and JFH1-CEtrans-S1/L/R, for

447 which the SHAPE reactivities suggested that the 5' and 3' stem mutations did not recapitulate

448 wild type SLVI as expected due to increased reactivity in the 5' basal stem, but neither were they

449 representative of the 'closed' conformation (Fig. 4C, 5B). We transfected Huh 7.5 cells with 500

450 ng of RNA of each template, in parallel, and normalised translation levels to a Renilla luciferase

451 transfection control RNA (Fig. 6B). As predicted for the 'open' conformation and blocking of

452 the LRA, JFH1-CEtrans-S1 showed a small, but significant, increase in translation level

453 compared to both JFH1-CEtrans and JFH1-CEtrans-S1/L ( $<<0.05)$. In contrast, JFH1-CEtrans-

$454 \mathrm{~S} 1 / \mathrm{L}$, shown to preferentially form the 'closed' structure, did not show the predicted reduction in

455 translation and was unchanged from parental JFH1-CEtrans. Similarly, despite small increases

456 and decreases in translation levels of JFH1-CEtrans-L/R and JFH1-CEtrans-S1/L/R respectively,

457 these were not significantly different when compared to the parental template. This suggests that,

458 even though the wild type SLVI structure is not fully restored, with reduced base pairing

459 remaining in the basal 5' stem, this did not impact translation.

460 The limited changes in phenotype observed strongly suggested that the difference

461 observed between JFH1-luc-trans: $\mathrm{NS} 5 \mathrm{~B}$ and JFH1-CEtrans is due to an as yet unidentified role 
462 for SLV and VI, and is not miR122 or LRA dependent. In the presence of miR122 both these 463 templates exhibit the 'open' conformation as determined by SHAPE analysis (Fig. 3A, 4A). Due 464 to the mutations introduced into JFH1-CEtrans-S1 only JFH1-CEtrans is capable of forming the 465 'closed' conformation which, in the absence of miR122, is the preferred conformation (Fig. 3B). 466 As the switch between the two conformations is miR122 dependent we reasoned that, if the 467 'closed' conformation is in fact responsible for the reduction in translation of JFH1-CEtrans, we 468 would observe a similar difference when comparing JFH1-CEtrans ('closed') to JFH1-CEtrans469 S1 ('open') in cells lacking miR122. We therefore investigated translation of JFH1-luc470 trans: $\triangle$ NS5B, JFH1-CEtrans, JFH1-CEtrans-S1 and JFH1-CEtrans-S1/L in HeLa cells, which 471 are naturally lacking in miR122 (Jopling et al. 2005) (Fig. 6C). Overall we observed translation 472 levels that were $\sim 10$-fold lower in HeLa cells than in Huh 7.5 cells. However, the relative 473 translation phenotypes remained the same, with a significant reduction in translation for JFH1474 CEtrans, and all other templates that contained the core-extended sequence. Additionally, no 475 significant changes were observed between JFH1-CEtrans and JFH1-CEtrans-S1, or JFH1476 CEtrans-S1/L, which is also expected to form the 'closed' conformation in the absence of 477 miR122. This supports our contention that formation of the LRA does not account for the change 478 in translation phenotype between JFH1-luc-trans: $\triangle$ NS5B and JFH1-CEtrans, and conclude that 479 although the presence of SLVI clearly reduces translation (Fig. 6A, C) - this is unrelated to the 480 LRA and any miR122 induced switch between the 'open' and 'closed' conformations.

481 With no observable link between translation levels and the conformations demonstrated 482 by SHAPE analysis we went on to investigate whether there were differences in replication 483 between our structurally modified templates. To achieve this we used a core-extended version of 484 a JFH-1 replicon, JFH1-CErep. Huh 7.5 cells were transfected with 500 ng of template RNA, 485 containing the same modifications as above, and replication recorded as luciferase activity over a $48645 \mathrm{~h}$ time course. Our results show that for JFH1-CErep-L/R, where mutations were engineered 487 solely within SLVI, replication was not significantly altered from the unmodified parental 488 replicon, in keeping with the results for translation (Fig. 6D, 6B). In contrast, mutants JFH1489 CErep-S1, JFH1-CErep-S1/L and JFH1-CErep-S1/L/R, in which miR122 binding to S1 is 490 blocked, all showed a $>10$-fold, highly significant reduction in the level of replication $(\mathrm{p}<0.01)$ 491 (Fig. 6D). This is entirely consistent with our current understanding that miR122 binding to the 492 5'UTR of HCV is important for HCV replication (Jangra et al. 2010; Jopling et al. 2005). The 
493 addition of modified S1-miR122 to the RNA transfection mix, to complement the S1 mutations

494 in the replicon, restored replication of JFH1-CErep-S1 to parental levels, demonstrating that the 495 observed reductions in replication are due entirely to disruption of miR122 binding and not a 496 consequence of possible changes in conformation promoted by the LRA (Fig. 6E).

497 These results demonstrate that, while miR122 binding has a profound effect on the 498 replication of the HCV genome, neither replication nor translation phenotypes are significantly 499 influenced by modifications to SLVI that preferentially form the 'open' or 'closed' 500 conformations of the LRA.

501

502 Discussion

503

504

How are the competing events of single-stranded, positive-sense, RNA virus translation 505 and replication separated? At least early in the replication cycle, before compartmentalization into membrane-bound replication vesicles, these must involve interaction of the translating ribosome or the viral polymerase with the same template. One strategy, typified by poliovirus, requires the accumulation of one or more viral translation products to initiate genome replication (Barton \& Flanegan 1997; Jurgens \& Flanegan 2003). In this case, ribonucleoprotein complexes form involving phylogenetically conserved RNA stem-loop structures. Since many positivesense single-stranded RNA viruses have small genomes, the adoption of higher-order structures - that may vary in conformation depending upon the environment or availability of interacting

513 proteins - effectively increases the level of control that can be exerted during the replication 514 cycle. In particular, structures capable of forming long-range interactions are of interest as they may have the capability to cyclize the genome (Alvarez et al. 2005) or - by adopting alternate conformation - riboswitches (Ooms et al. 2004; Shetty et al. 2013; Wang \& White 2007)

518 Tuplin et al. 2012) and a number of long-range interactions have been predicted within it (Fricke 519 et al. 2015), some having demonstrable involvement in important aspects of the HCV replication 520 cycle (Diviney et al. 2008; Romero-López et al. 2014; Romero-López \& Berzal-Herranz 2009; 521 Shetty et al. 2013; Tuplin et al. 2015; You \& Rice 2008). Of these, the long-range association 522 (LRA) between nts 23-31 encompassing miR122 seed site 1 in the 5'UTR, and complementary 523 nts 433-441 located in the 5' basal stem of SLVI within the core protein coding region 
524 (Beguiristain et al. 2005; Díaz-Toledano et al. 2009; García-Sacristán et al. 2015), may adopt

525 'open' and 'closed' conformations, and is predicted to modulate the switch between translation

526 and replication of the virus genome (Fig. 1A) (Kim et al. 2003). The 'open' conformation (i.e. no

527 LRA) is proposed to favour translation, whereas the 'closed' conformation restricts access of the

528 ribosome to sequences within the coding region implicated in genome translation, thereby

529 favouring replication.

530 To investigate the structure and function of the 'open' and 'closed' conformations in

531 greater detail we have mapped the native structure using SHAPE in the presence and absence of

532 miR122. We have additionally extensively mutagenised sequences implicated in formation of

533 both the 'open' and 'closed' conformations, mapped their structure by SHAPE and investigated

534 the resulting influence on translation and genome replication.

535 Although the strong stem-loop (SLI) in the 5'UTR confounded SHAPE interrogation of

536 sequences forming the S1 site of miR122 binding, those contributing to the basal stem of SLVI

537 were readily mapped. Having determined the influence on miR122 binding of mutations in the

538 S1 site (Fig. 2) we inferred the LRA and formation of the 'closed' structure from exposure or

539 otherwise of the basal stem of SLVI. The LRA was detectable only under very specific

540 conditions, including an in vitro assay in which miR122 was omitted. Similarly, mutagenesis of

541 the template within the S1 miR122 binding site (to prevent miR122 binding) and introduction of

542 complementary mutations to the 5 ' basal stem of SLVI allowed the LRA to be inferred. In

543 contrast, in the presence of miR122, and/or unmodified sequences at the basal stem of SLVI, we

544 were unable to detect the LRA and formation of the 'closed' structure. We propose that, under

545 conditions in which miR122 is present in significant amounts, the phylogenetically conserved

546 basal stem of SLVI is unlikely to separate to form a long-range association.

$547 \quad$ However, incorporation of 5' (L) and 3' (R) mutations to SLVI did lead to structural

548 changes within the stem-loop that were not predicted by mfold. With the exception of JFH1-

549 CEtrans-S1/L, which clearly adopts the 'closed' conformation, all the tested substitutions to the

550 basal stem of SLVI increased the NMIA-reactivity of the structure (Fig. 4 and 5), indicating a

551 reduction in complementary pairing that was more extensive than the sites of modification. In

552 addition, when not paired with the 5' mutations, the 3' mutants (JFH1-CEtrans-R and JFH1-

553 CEtrans-S1/R) showed further modification of the SLVI structure with the loss of reactivity of

554 nts 500-501 (Fig. 5A and 4D). In these cases, it is clear that SLVI had undergone more extensive 
555 alteration of base pairing and structure. Previous studies of SLVI, independent of LRA

556 disruption, were shown to result in alteration to the translation phenotype (Vassilaki et al. 2008).

557 Without a greater understanding of the RNA structure in this region, for example by expanding

558 the region analysed by SHAPE mapping, we do not think a complete interpretation of the

559 relationship between RNA structure and phenotype is possible. In addition, the HCV IRES is

560 known to be flexible and dynamic (Pérard et al. 2013) and it is possible that the averaged data

561 obtained from SHAPE mapping may obscure some of this flexibility. Indeed, the error bars

562 covering some data points may suggest a much more dynamic situation at the single molecule

563 level than is represented by the overall RNA population while in solution.

564 Notwithstanding the potential for the LRA forming during HCV infection in vivo, we

565 went on to analyse $\mathrm{HCV}$ translation and replication from templates in which we engineered the

566 'open' or 'closed' conformations by mutagenesis of the complementary pairing critical for LRA

567 formation. We reasoned that, if dramatic differences were observable, this might indicate a role

568 for the LRA and our ability to detect the 'closed' structure in vitro might not be representative of

569 conditions in vivo. For example, cellular proteins could influence the formation of the LRA or

570 miR122 may be limiting or absent at certain stages of the replication cycle.

571 We investigated translation from a core-extended template transfected into Huh 7.5 cells.

572 As expected from previous studies (Kim et al. 2003), extension of core-encoding sequences - to

573 encompass SLVI - reduced reporter gene expression by $\sim 50 \%$. When normalised to this lower

574 level of translation, the template engineered to adopt the 'closed' conformation (JFH1-CEtrans-

$575 \mathrm{~S} 1 / \mathrm{L}$ ) exhibited levels of translation essentially indistinguishable from the control. In contrast,

576 the template unable to recruit miR122 to the S1 seed site (JFH1-CEtrans-S1) and therefore solely

577 adopting the 'open' conformation, exhibited a slight but significant increase in translation (Fig.

578 6B). Compared to its vital role in viral replication, the role of miR122 in stimulating HCV

579 translation is not as clear, with some reports suggesting mutations in miR122 seed sites do not

580 lead to changes in translation (Jopling et al. 2005) and others observing decreases in translation

581 upon mutation of either, or both, seed sites (Jangra et al. 2010). Our results for JFH1-CEtrans-S1

582 and JFH1-CEtrans-S1/L/R, both adopting an 'open' conformation, would support the studies by

583 Jopling and colleagues (Jopling et al. 2005). Despite inhibition of miR122 binding at the S1 site,

584 both templates were still able to recruit miR122 to the S2 site (Fig. 2), thus maintaining parental,

585 or near-parental levels of translation. Therefore, templates of known conformation with regard to 
586 the LRA did not show a correlation between a closed structure and a reduction in translation, and 587 opening the structure only led to a minor increase in translation in one template, JFH1-CEtrans-

588 S1. In addition, relative translation phenotypes in HeLa cells were comparable to Huh 7.5 cells 589 demonstrating that the presence of SLVI is sufficient to account for the reduction in translation 590 observed between JFH1-luc-trans: $\triangle$ NS5B and JFH1-CEtrans (Fig. 6B, C). The presence of SLVI 591 has also been shown to influence translation through modulation of RNA interactions involving 592 domain 5BSL3.2 (Ventura et al. 2017). Such interactions may contribute to the translation 593 phenotypes we have demonstrated here to be independent of the LRA.

594 In contrast to the results obtained using translation templates bearing mutations to 595 destroy/recreate the LRA, analysis of genome replication bearing identical mutations was easier 596 to interpret. In these, any mutation of the S1 miR122 seed site reduced replication by $\sim 2 \log _{10}$ at $59728 \mathrm{~h}$ post-transfection (Fig. 6C) and this phenotype could be readily and fully rescued by 598 provision of a complementary S1-miR122 in trans. These results imply that the structures 599 adopted by sequences predicted to be involved in the LRA either have no influence on genome 600 replication, or that the influence is negligible when compared to the known impact of reduced 601 miR122 binding (Jopling et al. 2008; Jopling et al. 2005; Li et al. 2013).

602 Taken together, these studies suggest that the LRA between the S1 binding site and the 603 basal stem of SLVI is unlikely to contribute to temporal control of genome translation and 604 replication. In the absence of introduced complementary mutations we could only demonstrate 605 formation of the LRA-associated 'closed' structure under very specific conditions in which 606 miR122 was absent (Fig. 3B). Whether such conditions occur in vivo is unclear. An estimated 607 66,000 copies of miR122 have been reported in hepatocytes (Jopling 2012) and Luna et al., 608 (Luna et al. 2015) have reported that HCV replication de-represses cellular targets of miR122, 609 implying that the replicating virus genome acts as a 'sponge' to sequester miR122. Although the 610 latter suggests that miR122 is limiting, it is unlikely to be early in the infection cycle. At this 611 time a small number of genomes are present and temporal control of genome translation and 612 replication is likely critical outside the compartmentalisation offered by membrane-bound 613 replication complexes (Wölk et al. 2008) (Miyanari et al. 2003).

614

\section{Conclusions}

616 
618 modulate essential genome functions such as translation and replication. In this study we demonstrate that one such conformational change, the LRA, involving complementary sequences

620 in the HCV IRES and a core gene stem-loop structure, is unlikely to act as a modulator between

621 translation and replication. We have shown that switching between the 'open' and 'closed'

622 conformations is a miR122 dependent process and confirmed that presence of the core stem-loop

623 structure SLVI results in a drop in translation activity. However we have demonstrated that

624 templates preferentially forming either the 'open' or 'closed' conformation are not associated

625 with any translation or replication phenotypes. Instead we propose that the stem-loop structure

626 SLVI, mediates translation via other, as yet undefined mechanisms.

627

628

$\underline{\text { References }}$

629

Adams RL, Pirakitikulr N, and Pyle AM. 2017. Functional RNA structures throughout the Hepatitis C Virus genome. Curr Opin Virol 24:79-86. 10.1016/j.coviro.2017.04.007

Alvarez DE, Lodeiro MF, Ludueña SJ, Pietrasanta LI, and Gamarnik AV. 2005. Long-range RNA-RNA interactions circularize the dengue virus genome. J Virol 79:6631-6643. 79/11/6631 [pii]

10.1128/JVI.79.11.6631-6643.2005

Barton DJ, and Flanegan JB. 1997. Synchronous replication of poliovirus RNA: initiation of negative-strand RNA synthesis requires the guanidine-inhibited activity of protein 2C.J Virol 71:8482-8489.

Beguiristain N, Robertson HD, and Gómez J. 2005. RNase III cleavage demonstrates a long range RNA: RNA duplex element flanking the hepatitis $\mathrm{C}$ virus internal ribosome entry site. Nucleic Acids Res 33:5250-5261. 10.1093/nar/gki822

Chambers TJ, Hahn CS, Galler R, and Rice CM. 1990. Flavivirus genome organization, expression, and replication. Annu Rev Microbiol 44:649-688. 10.1146/annurev.mi.44.100190.003245

Diviney S, Tuplin A, Struthers M, Armstrong V, Elliott RM, Simmonds P, and Evans DJ. 2008. A hepatitis $C$ virus cis-acting replication element forms a long-range RNA-RNA interaction with upstream RNA sequences in NS5B. J Virol 82:9008-9022. 10.1128/JVI.02326-07

Díaz-Toledano R, Ariza-Mateos A, Birk A, Martínez-García B, and Gómez J. 2009. In vitro characterization of a miR-122-sensitive double-helical switch element in the 5 ' region of hepatitis C virus RNA. Nucleic Acids Res 37:5498-5510. gkp553 [pii]

10.1093/nar/gkp553

Fricke M, Dünnes N, Zayas M, Bartenschlager R, Niepmann M, and Marz M. 2015. Conserved RNA secondary structures and long-range interactions in hepatitis C viruses. RNA 21:1219-1232. 10.1261/rna.049338.114 
656 Friebe P, Lohmann V, Krieger N, and Bartenschlager R. 2001. Sequences in the 5 '

657

658

659

660

661

662

663

664

665

666

667

668

669

670

671

672

673

674

675

676

677

678

679

680

681

682

683

684

685

686

687

688

689

690

691

692

693

694

695

696

697

698

699

700

701

nontranslated region of hepatitis C virus required for RNA replication. Journal of Virology 75:12047-12057.

Gao M, Nettles RE, Belema M, Snyder LB, Nguyen VN, Fridell RA, Serrano-Wu MH, Langley DR, Sun JH, O'Boyle DR, Lemm JA, Wang C, Knipe JO, Chien C, Colonno RJ, Grasela DM, Meanwell NA, and Hamann LG. 2010. Chemical genetics strategy identifies an HCV NS5A inhibitor with a potent clinical effect. Nature 465:96-100. 10.1038 /nature08960

García-Sacristán A, Moreno M, Ariza-Mateos A, López-Camacho E, Jáudenes RM, Vázquez L, Gómez J, Martín-Gago J, and Briones C. 2015. A magnesium-induced RNA conformational switch at the internal ribosome entry site of hepatitis $\mathrm{C}$ virus genome visualized by atomic force microscopy. Nucleic Acids Res 43:565-580. 10.1093/nar/gku1299

Honda M, Rijnbrand R, Abell G, Kim D, and Lemon SM. 1999. Natural variation in translational activities of the $5^{\prime}$ nontranslated RNAs of hepatitis $\mathrm{C}$ virus genotypes $1 \mathrm{a}$ and $1 \mathrm{~b}$ : evidence for a long-range RNA-RNA interaction outside of the internal ribosomal entry site. J Virol 73:4941-4951.

Jangra RK, Yi M, and Lemon SM. 2010. Regulation of hepatitis C virus translation and infectious virus production by the microRNA miR-122. J Virol 84:6615-6625. JVI.00417-10 [pii]

10.1128/JVI.00417-10

Jopling C. 2012. Liver-specific microRNA-122: Biogenesis and function. RNA Biol 9:137-142. 10.4161/rna.18827

Jopling CL, Schütz S, and Sarnow P. 2008. Position-dependent function for a tandem microRNA miR-122-binding site located in the hepatitis C virus RNA genome. Cell Host Microbe 4:77-85. S1931-3128(08)00173-X [pii]

10.1016/j.chom.2008.05.013

Jopling CL, Yi M, Lancaster AM, Lemon SM, and Sarnow P. 2005. Modulation of hepatitis C virus RNA abundance by a liver-specific MicroRNA. Science 309:1577-1581. $309 / 5740 / 1577$ [pii]

$10.1126 /$ science. 1113329

Jurgens C, and Flanegan JB. 2003. Initiation of poliovirus negative-strand RNA synthesis requires precursor forms of $\mathrm{p} 2$ proteins. J Virol 77:1075-1083.

Kim YK, Lee SH, Kim CS, Seol SK, and Jang SK. 2003. Long-range RNA-RNA interaction between the 5 ' nontranslated region and the core-coding sequences of hepatitis $\mathrm{C}$ virus modulates the IRES-dependent translation. RNA 9:599-606.

Kuiken C, Combet C, Bukh J, Shin-I T, Deleage G, Mizokami M, Richardson R, Sablon E, Yusim K, Pawlotsky JM, Simmonds P, and Los Alamos HIVdg. 2006. A comprehensive system for consistent numbering of HCV sequences, proteins and epitopes. Hepatology 44:1355-1361. 10.1002/hep.21377

Lagos-Quintana M, Rauhut R, Yalcin A, Meyer J, Lendeckel W, and Tuschl T. 2002. Identification of tissue-specific microRNAs from mouse. Curr Biol 12:735-739.

Lawitz E, Lalezari JP, Hassanein T, Kowdley KV, Poordad FF, Sheikh AM, Afdhal NH, Bernstein DE, Dejesus E, Freilich B, Nelson DR, Dieterich DT, Jacobson IM, Jensen D, Abrams GA, Darling JM, Rodriguez-Torres M, Reddy KR, Sulkowski MS, Bzowej NH, Hyland RH, Mo H, Lin M, Mader M, Hindes R, Albanis E, Symonds WT, Berrey MM, and Muir A. 2013.

PeerJ reviewing PDF | (2018:07:29568:1:1:NEW 13 Sep 2018) 
702

703

704

705

706

707

708

709

710

711

712

713

714

715

716

717

718

719

720

721

722

723

724

725

726

727

728

729

730

731

732

733

734

735

736

737

738

739

740

741

742

743

744

745

746

Sofosbuvir in combination with peginterferon alfa-2a and ribavirin for non-cirrhotic, treatment-naive patients with genotypes 1,2 , and 3 hepatitis $\mathrm{C}$ infection: a randomised, double-blind, phase 2 trial. Lancet Infect Dis 13:401-408. 10.1016/S1473-3099(13)70033-1

Li Y, Masaki T, Yamane D, McGivern DR, and Lemon SM. 2013. Competing and noncompeting activities of miR-122 and the 5' exonuclease Xrn1 in regulation of hepatitis C virus replication. Proc Natl Acad Sci U S A 110:1881-1886. 10.1073/pnas.1213515110

Li Z, and Nagy PD. 2011. Diverse roles of host RNA binding proteins in RNA virus replication. RNA Biol 8:305-315.

Lindenbach BD, Evans MJ, Syder AJ, Wolk B, Tellinghuisen TL, Liu CC, Maruyama T, Hynes RO, Burton DR, McKeating JA, and Rice CM. 2005. Complete replication of hepatitis C virus in cell culture. Science 309:623-626.

Luna JM, Scheel TK, Danino T, Shaw KS, Mele A, Fak JJ, Nishiuchi E, Takacs CN, Catanese MT, de Jong YP, Jacobson IM, Rice CM, and Darnell RB. 2015. Hepatitis C virus RNA functionally sequesters miR-122. Cell 160:1099-1110. 10.1016/j.cell.2015.02.025

Mauger DM, Golden M, Yamane D, Williford S, Lemon SM, Martin DP, and Weeks KM. 2015. Functionally conserved architecture of hepatitis C virus RNA genomes. Proc Natl Acad Sci U S A 112:3692-3697. 10.1073/pnas.1416266112

McMullan LK, Grakoui A, Evans MJ, Mihalik K, Puig M, Branch AD, Feinstone SM, and Rice CM. 2007. Evidence for a functional RNA element in the hepatitis $C$ virus core gene. Proc Natl Acad Sci U S A 104:2879-2884. 0611267104 [pii]

10.1073/pnas.0611267104

Merino EJ, Wilkinson KA, Coughlan JL, and Weeks KM. 2005. RNA structure analysis at single nucleotide resolution by selective 2'-hydroxyl acylation and primer extension (SHAPE). J Am Chem Soc 127:4223-4231. 10.1021/ja043822v

Miyanari Y, Hijikata M, Yamaji M, Hosaka M, Takahashi H, and Shimotohno K. 2003. Hepatitis $\mathrm{C}$ virus non-structural proteins in the probable membranous compartment function in viral genome replication. J Biol Chem 278:50301-50308. 10.1074/jbc.M305684200

Mohd Hanafiah K, Groeger J, Flaxman AD, and Wiersma ST. 2013. Global epidemiology of hepatitis C virus infection: new estimates of age-specific antibody to HCV seroprevalence. Hepatology 57:1333-1342.10.1002/hep.26141

Mortimer SA, and Doudna JA. 2013. Unconventional miR-122 binding stabilizes the HCV genome by forming a trimolecular RNA structure. Nucleic Acids Res 41:4230-4240. $10.1093 /$ nar/gkt075

Nicholson BL, and White KA. 2014. Functional long-range RNA-RNA interactions in positivestrand RNA viruses. Nat Rev Microbiol 12:493-504. 10.1038/nrmicro3288

Niepmann M, Shalamova LA, Gerresheim GK, and Rossbach 0. 2018. Signals Involved in Regulation of Hepatitis C Virus RNA Genome Translation and Replication. Front Microbiol 9:395. 10.3389/fmicb.2018.00395

Ooms M, Huthoff H, Russell R, Liang C, and Berkhout B. 2004. A riboswitch regulates RNA dimerization and packaging in human immunodeficiency virus type 1 virions. J Virol 78:10814-10819. 10.1128/JVI.78.19.10814-10819.2004

Pang PS, Pham EA, Elazar M, Patel SG, Eckart MR, and Glenn JS. 2012. Structural map of a microRNA-122: hepatitis C virus complex. J Virol 86:1250-1254. JVI.06367-11 [pii]

10.1128/JVI.06367-11 
747 Pérard J, Leyrat C, Baudin F, Drouet E, and Jamin M. 2013. Structure of the full-length HCV

748

749

750

751

752

753

754

755

756

757

758

759

760

761

762

763

764

765

766

767

768

769

770

771

772

773

774

775

776

777

778

779

780

781

782

783

784

785

786

787

788

789

790 IRES in solution. Nat Commun 4:1612.10.1038/ncomms2611

Reuter JS, and Mathews DH. 2010. RNAstructure: software for RNA secondary structure prediction and analysis. BMC Bioinformatics 11:129. 10.1186/1471-2105-11-129

Reynolds JE, Kaminski A, Kettinen HJ, Grace K, Clarke BE, Carroll AR, Rowlands DJ, and Jackson RJ. 1995. Unique features of internal initiation of hepatitis C virus RNA translation. EMBO J 14:6010-6020.

Romero-López C, Barroso-Deljesus A, García-Sacristán A, Briones C, and Berzal-Herranz A. 2014. End-to-end crosstalk within the hepatitis $C$ virus genome mediates the conformational switch of the 3'X-tail region. Nucleic Acids Res 42:567-582. $10.1093 /$ nar/gkt841

Romero-López C, and Berzal-Herranz A. 2009. A long-range RNA-RNA interaction between the 5' and 3' ends of the HCV genome. RNA 15:1740-1752. rna.1680809 [pii]

10.1261/rna.1680809

Sedano CD, and Sarnow P. 2014. Hepatitis C virus subverts liver-specific miR-122 to protect the viral genome from exoribonuclease Xrn2. Cell Host Microbe 16:257-264. 10.1016/j.chom.2014.07.006

Shetty S, Stefanovic S, and Mihailescu MR. 2013. Hepatitis C virus RNA: molecular switches mediated by long-range RNA-RNA interactions? Nucleic Acids Res 41:2526-2540. $10.1093 /$ nar/gks1318

Shimakami T, Yamane D, Jangra RK, Kempf BJ, Spaniel C, Barton DJ, and Lemon SM. 2012. Stabilization of hepatitis C virus RNA by an Ago2-miR-122 complex. Proc Natl Acad Sci U S A 109:941-946. 10.1073/pnas.1112263109

Simmonds P, Tuplin A, and Evans DJ. 2004. Detection of genome-scale ordered RNA structure (GORS) in genomes of positive-stranded RNA viruses: Implications for virus evolution and host persistence. RNA 10:1337-1351. 10.1261/rna.7640104

Tuplin A. 2015. Diverse roles and interactions of RNA structures during the replication of positive-stranded RNA viruses of humans and animals. J Gen Virol 96:1497-1503. 10.1099/vir.0.000066

Tuplin A, Evans DJ, and Simmonds P. 2004. Detailed mapping of RNA secondary structures in core and NS5B-encoding region sequences of hepatitis C virus by RNase cleavage and novel bioinformatic prediction methods. J Gen Virol 85:3037-3047. 10.1099/vir.0.80141-0

Tuplin A, Struthers M, Cook J, Bentley K, and Evans DJ. 2015. Inhibition of HCV translation by disrupting the structure and interactions of the viral CRE and 3' X-tail. Nucleic Acids Res 43:2914-2926. 10.1093/nar/gkv142

Tuplin A, Struthers M, Simmonds P, and Evans DJ. 2012. A twist in the tail: SHAPE mapping of long-range interactions and structural rearrangements of RNA elements involved in HCV replication. Nucleic Acids Res 40:6908-6921. 10.1093/nar/gks370

Vassilaki N, Friebe P, Meuleman P, Kallis S, Kaul A, Paranhos-Baccalà G, Leroux-Roels G, Mavromara P, and Bartenschlager R. 2008. Role of the hepatitis C virus core+1 open reading frame and core cis-acting RNA elements in viral RNA translation and replication. J Virol 82:11503-11515. JVI.01640-08 [pii]

10.1128/JVI.01640-08 
791 Ventura M, Martin L, Jaubert C, Andréola ML, and Masante C. 2017. Hepatitis C virus 792 793

794 795

801

802

803

804

805

806

807

808 intragenomic interactions are modulated by the SLVI RNA structure of the core coding sequence. J Gen Virol 98:633-642. 10.1099/jgv.0.000719

Wang S, and White KA. 2007. Riboswitching on RNA virus replication. Proc Natl Acad Sci U S A 104:10406-10411. 10.1073/pnas.0704178104

Welzel TM, Nelson DR, Morelli G, Di Bisceglie A, Reddy RK, Kuo A, Lim JK, Darling J, Pockros P, Galati JS, Frazier LM, Alqahtani S, Sulkowski MS, Vainorius M, Akushevich L, Fried MW, Zeuzem S, and Group H-TS. 2017. Effectiveness and safety of sofosbuvir plus ribavirin for the treatment of HCV genotype 2 infection: results of the real-world, clinical practice HCV-TARGET study. Gut 66:1844-1852. 10.1136/gutjnl-2016311609

Wölk B, Büchele B, Moradpour D, and Rice CM. 2008. A dynamic view of hepatitis C virus replication complexes. J Virol 82:10519-10531. 10.1128/JVI.00640-08

You S, and Rice CM. 2008. 3' RNA elements in hepatitis C virus replication: kissing partners and long poly(U). J Virol 82:184-195.

Zuker M. 2003. Mfold web server for nucleic acid folding and hybridization prediction. Nucleic Acids Res 31:3406-3415.

809

810

811 


\section{Figure 1 (on next page)}

Schematics of RNA structures and templates used.

(A) The predicted 'open' and 'closed' conformations of the HCV 5'UTR from SL I-VI with the addition, or loss, of miR122 as shown. S1 and S2 highlight the known binding sites of miR122. Black arrows indicate position and directionality of SHAPE primers. The red box shows expanded view of nucleotides 1-42 of JFH-1 with miR122 (lower case) bound to sites S1 and S2, with the blue box showing expanded view of nucleotides 428-507 of JFH-1 encompassing SLVI. Nucleotides in red are those predicted to be directly involved in formation of the LRA and mutations are indicated by feint black arrows indicating the substitutions made. Figure recreated and adapted from (Díaz-Toledano et al. 2009) . (B) The core-extended JFH-1 bicistronic translation reporter (top) and replicon (bottom). (C) The miR122 binding sites and SLVI with 5' stem (L), 3' stem (R) and S1 mutations displayed. The predicted blocking of miR122 binding, or SLVI formation, of each pair of mutations is shown. Black arrows represent the relative likely conformation, 'open' or 'closed', predicted to be favoured by each template. 


\section{A}

'Closed'

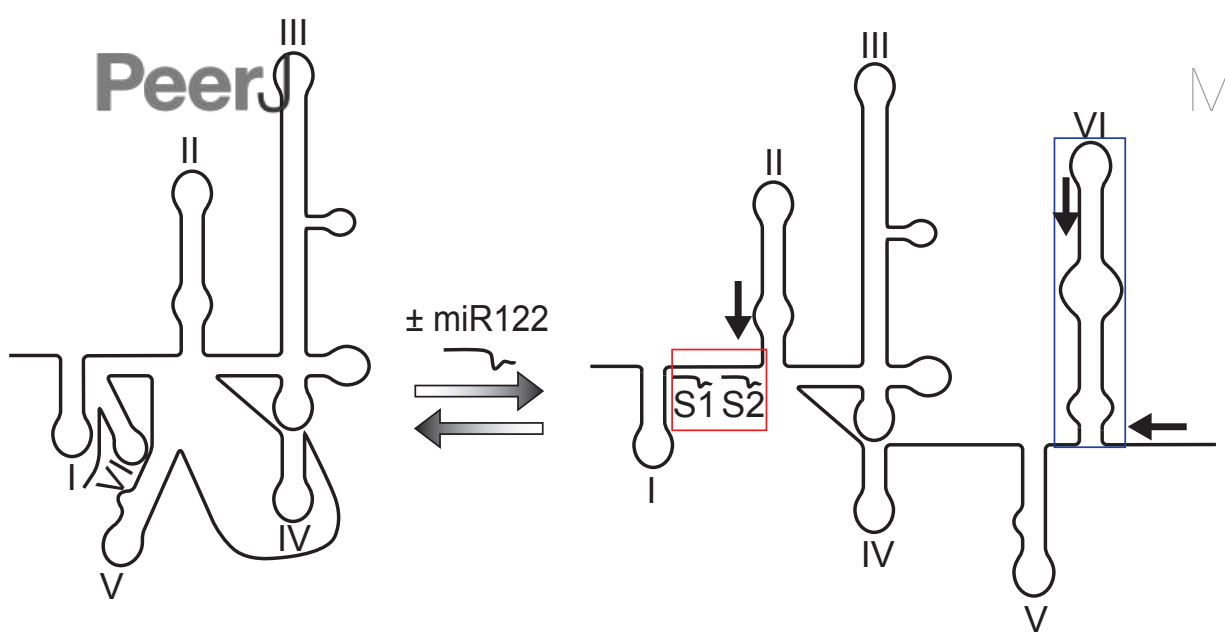

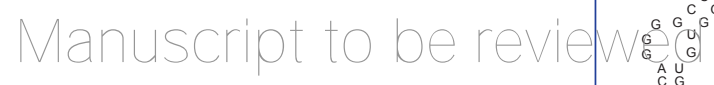

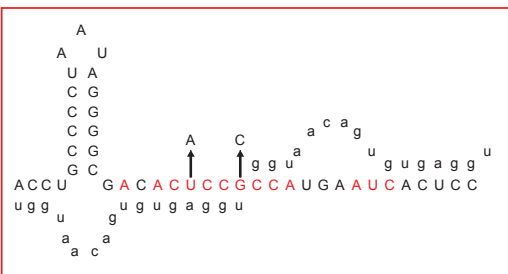

B

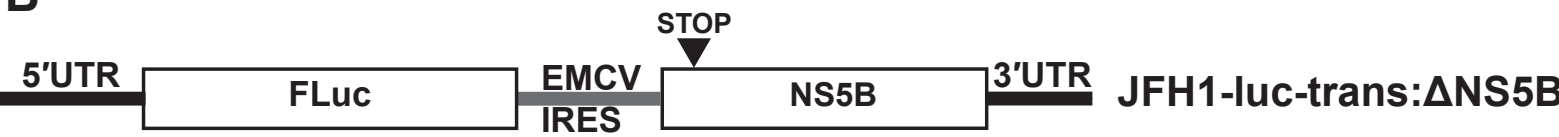

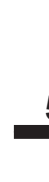

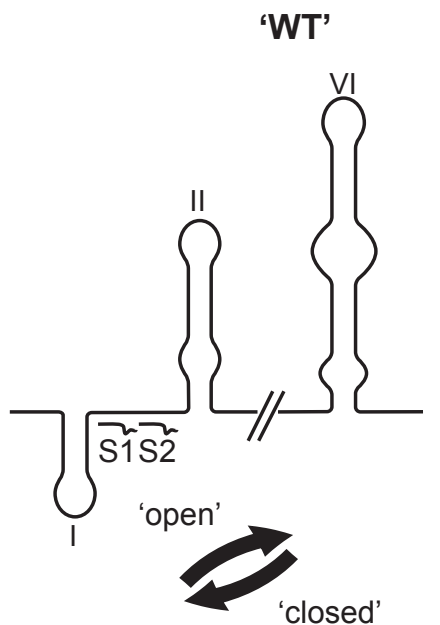

'S1'

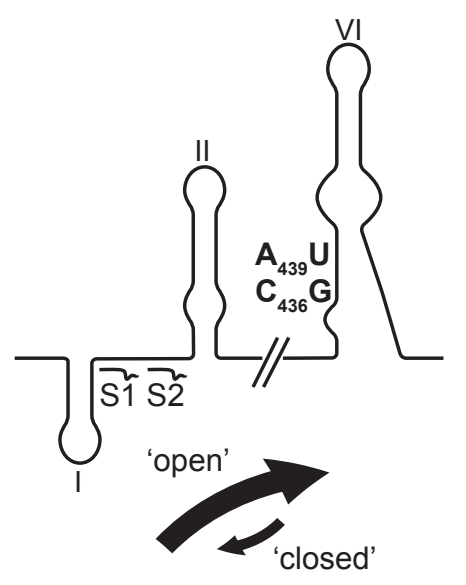

'S1/L'
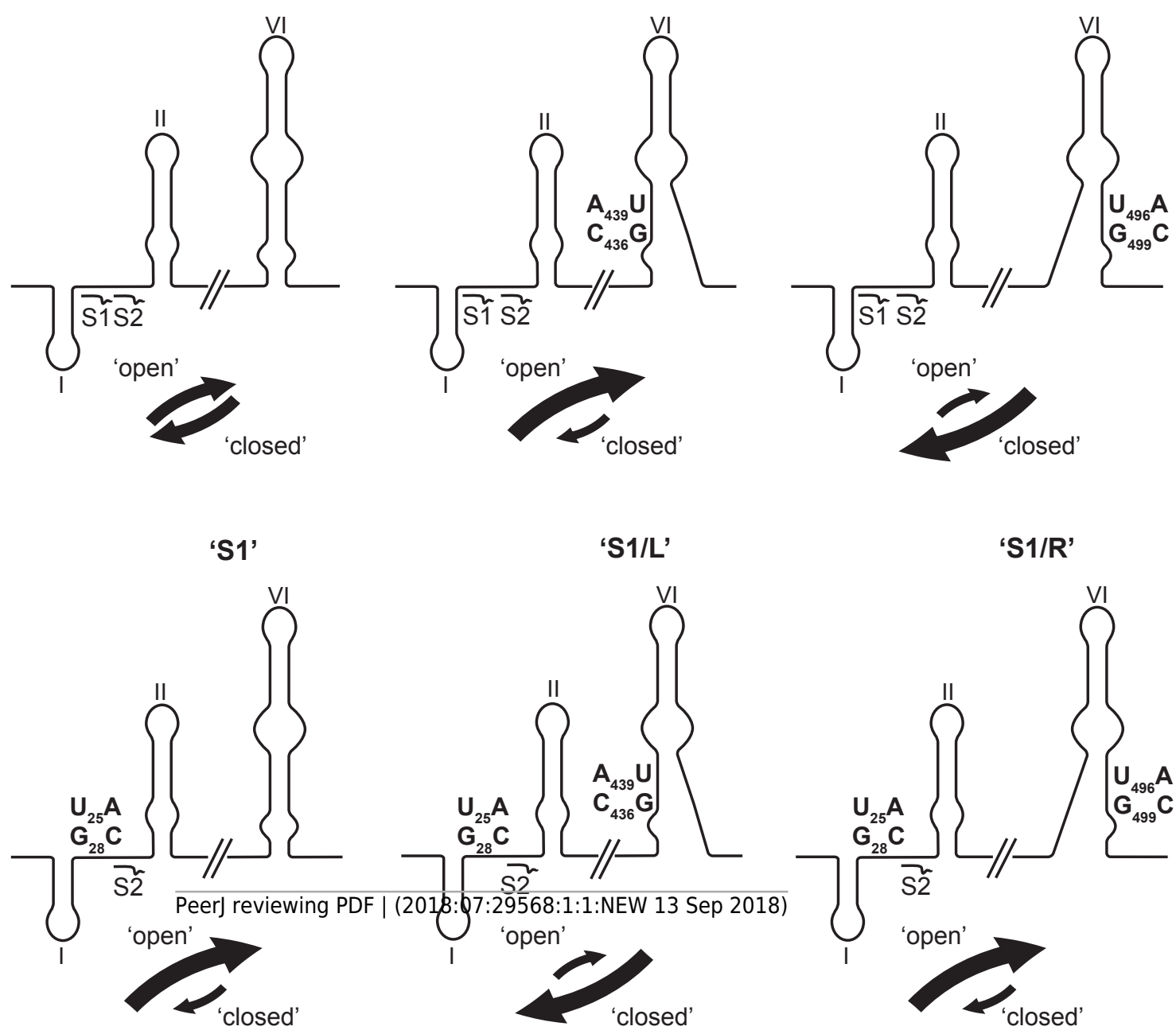

'S1/R'
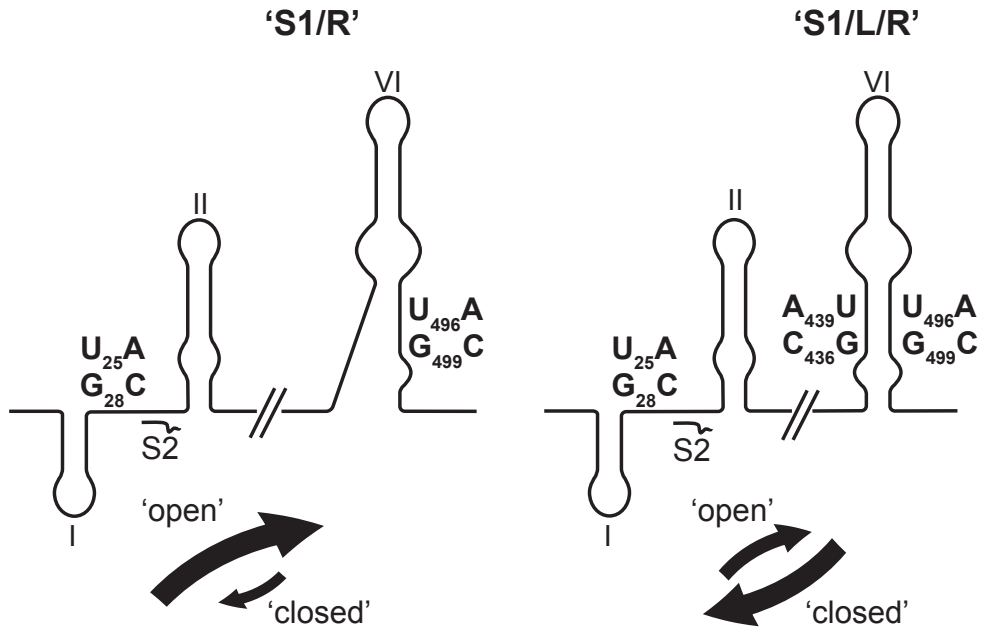
Figure 2 (on next page)

RNA-RNA electrophoretic mobility gel shift assays of miR122 binding to JFH-1 5'UTR.

A synthetic RNA of nts 1-50 of JFH-1 (JFH1 $\left.1^{1-50}\right)$ was complexed with increasing molar ratios of (A) wild type or (B) antisense synthetic miR122 and separated by non-denaturing PAGE. JFH1 $1^{1-50}$ mutated at the S1 binding site was similarly complexed with (C) wild type or (D) wild type plus an S1-mutated miR122 and separated by non-denaturing PAGE. miR122 binding was denoted by $1(+$ miR122) or $2(++$ miR122) reductions in RNA mobility compared to JFH1 $1^{1-50}$ control with no miR122 present (lane $\left.1, A-D\right)$. 
PeerJ

\section{$\begin{array}{lllllll}0 & 0.5 & 1 & 1.5 & 2 & 3 & 5\end{array}$

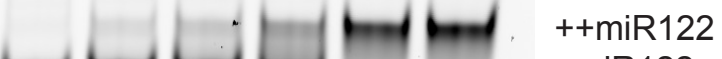 \\ + miR122}

JFH1 ${ }^{1-50}$

$\operatorname{miR} 122$

$\begin{array}{lllllll}0 & 0.5 & 1 & 1.5 & 2 & 3 & 5\end{array}$

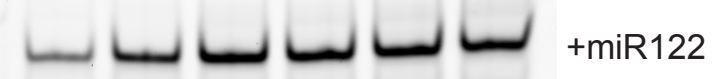

S1-JFH1 1-50

miR122
$\mathbf{B}$ anuscript to be reviewed

$\begin{array}{lllllll}0 & 0.5 & 1 & 1.5 & 2 & 3 & 5\end{array}$

$\mathrm{JFH} 1^{1-50}$

miR122-Comp

D

$\begin{array}{lllllll}0 & 0.5 & 1 & 1.5 & 2 & 3 & 5\end{array}$

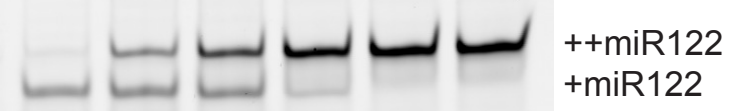

S1-JFH1 $1^{1-50}$

miR122 +

S1-miR122 


\section{Figure 3 (on next page)}

SHAPE analysis of parental template plus/minus miR122.

SHAPE reactivities are shown for (A) JFH1-CEtrans plus miR122, (B) JFH1-CEtrans minus miR122, and (C) JFH1-CEtrans plus LNA J22, with predicted (P) and observed (O) conformations given top right below template name. Black bars show normalised SHAPE reactivities of nucleotides 427-447 and 487-507, encompassing the $5^{\prime}$ and $3^{\prime}$ basal stems of SLVI respectively. Nucleotides with a reactivity of $<0.4$ are considered unreactive and therefore base-paired. Shaded regions highlight nucleotides of importance in determining 'open' or 'closed' conformations: specifically the $5^{\prime} \mathrm{G}_{434} \mathrm{G}_{435}$ motif and 3' nucleotides 494-507. The superimposed red line indicates the exposure of JFH1-CEtrans plus miR122, and is included on all plots for comparison of reactivities between a demonstrated 'open' conformation and the observed reactivity of additional templates. A maximum negative reactivity was set at -0.1 . Unless otherwise stated, error bars represent the SD of a minimum of 2 independent gel analyses for 2 replicate RNA-NMIA folding reactions. Figure $3 c$ was derived from only one replicate folding reaction. 

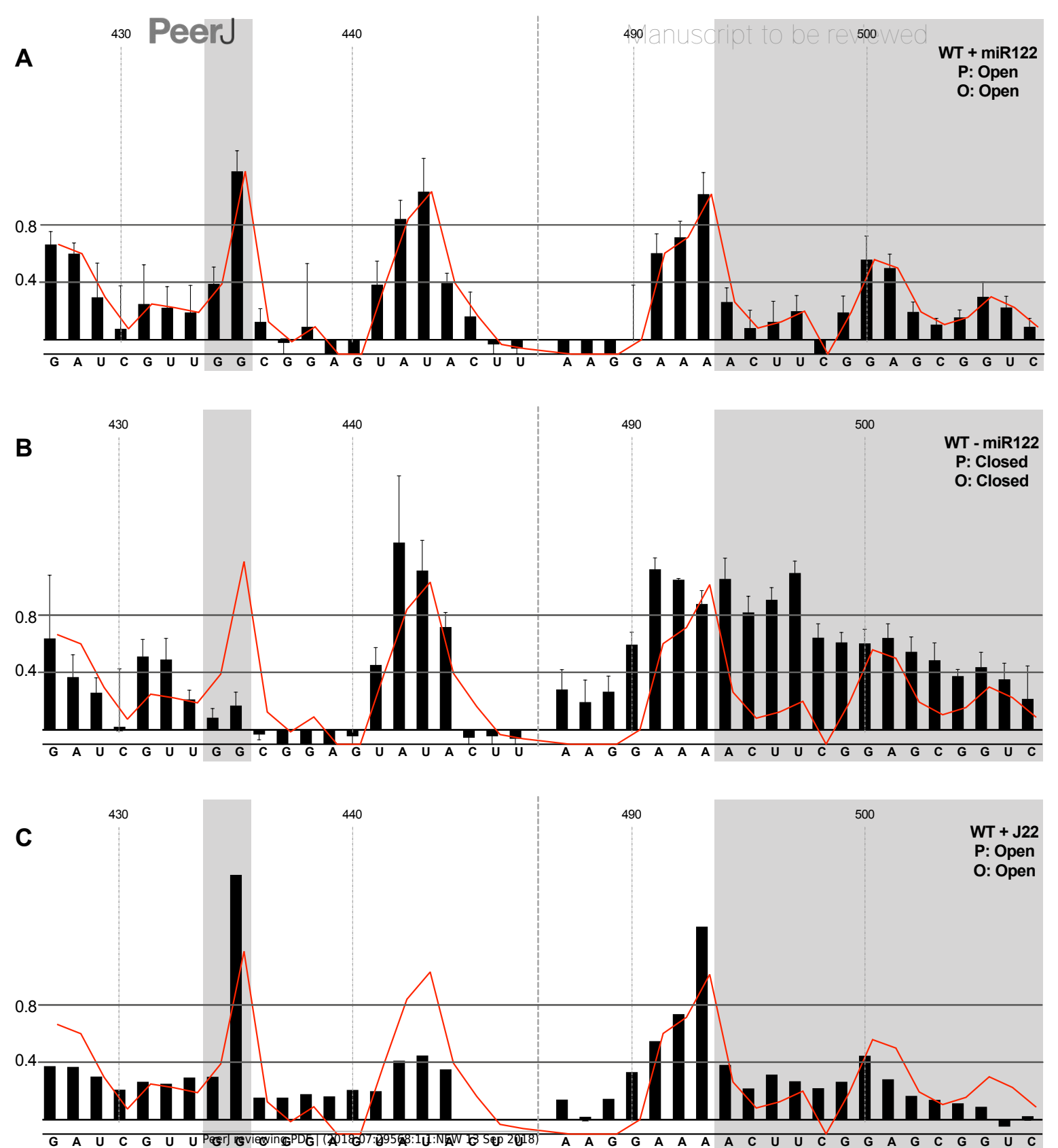
Figure 4(on next page)

SHAPE analysis of S1 and SLVI mutants with predicted 'open' conformation.

SHAPE reactivities are shown for (A) JFH1-CEtrans-S1, (B) JFH1-CEtrans-L, (C) JFH1-CEtrans$L / R$, and (D) JFH1-CEtrans-S1/R, with predicted (P) and observed (O) conformations given top right below template name. Data presentation as described in Figure 3. 
Figure $\mathbf{5}$ (on next page)

SHAPE analysis of S1 and SLVI mutants with predicted 'closed' conformation.

SHAPE reactivities are shown for (A) JFH1-CEtrans-S1/L, (B) JFH1-CEtrans-R, and (C) JFH1CEtrans-SI/L/R, with predicted $(\mathrm{P})$ and observed $(\mathrm{O})$ conformations given top right below template name. Data presentation as described in Figure 3. 

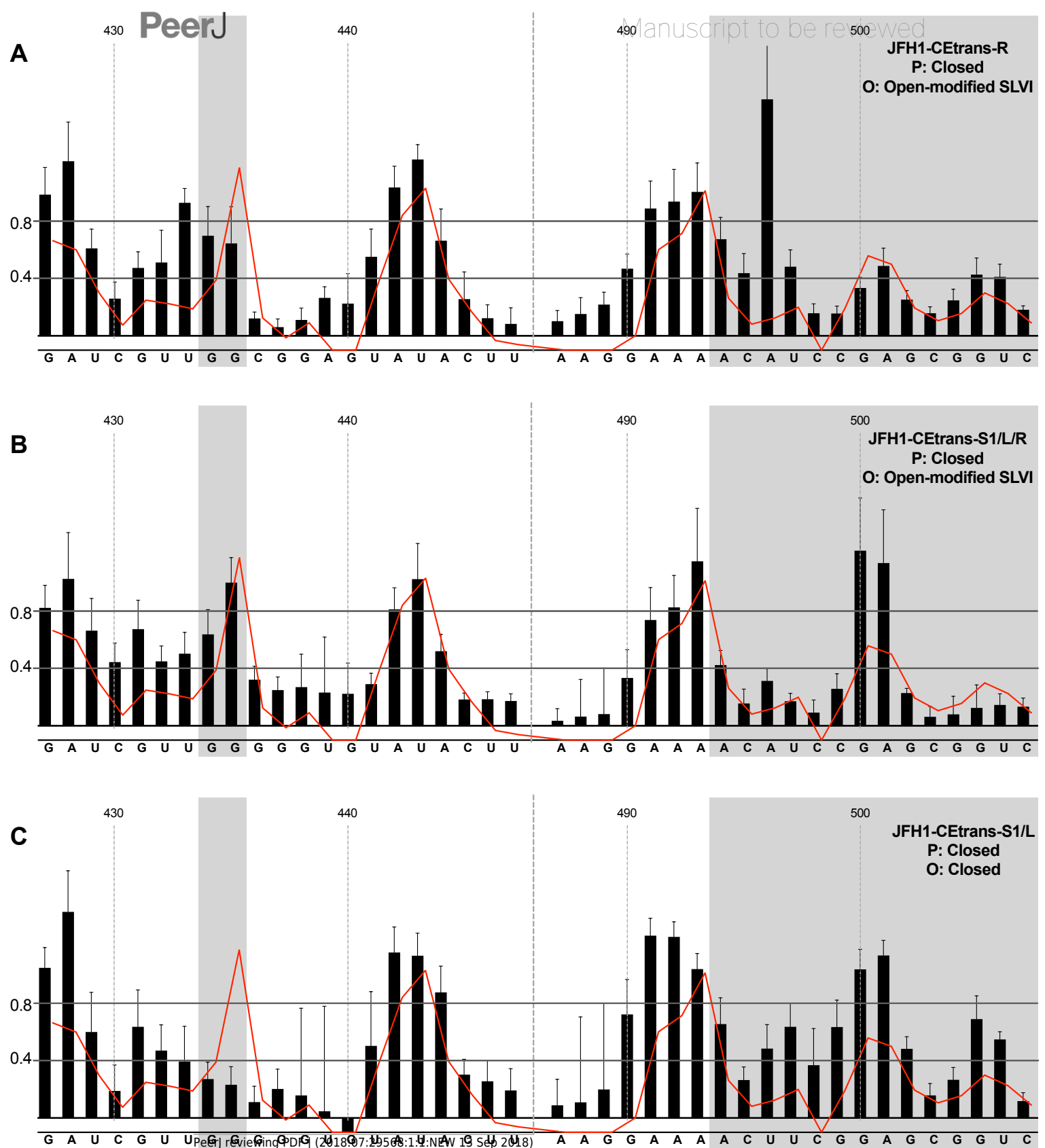


\section{Figure 6 (on next page)}

Phenotypic characterisation of JFH-1 reporter bearing S1 and SLVI mutations.

Translation levels were determined by luciferase assay for (A) JFH1-luc-trans:ANS5B and JFH1-CEtrans, (B) JFH1-CEtrans, S1 and SLVI mutants, and (C) JFH1-luc-trans:ANS5B and JFH1CEtrans in HeLa cells. Cell lysates were harvested at $4 \mathrm{~h}$ and luciferase readings calculated as a ratio of Firefly luciferase to a co-transfected Renilla luciferase control RNA. Replication kinetics of (D) JFH1-CErep, S1 and SLVI mutants, and (E) JFH1-CErep-S1 supplemented with S1-miR122, were determined by luciferase assays at 4, 21, 28, and 45h post-transfection. Luciferase readings are expressed as a percentage of the $4 \mathrm{~h}$ reading to normalise against translation of input RNA. A polymerase active site mutant, GDD to GNN, was included as replication control (Pol -ve). For all assays error bars represent SD of 3 replicate transfections from triplicate experiments, with statistical significance calculated by unpaired t-test analysis using GraphPad Prism V7. 
Table $\mathbf{1}$ (on next page)

Substitutions and predicted conformations 
1 Table 1 - Substitutions and predicted conformations

2

\begin{tabular}{lll} 
Mutant & Mutations & Predicted \\
& & Conformation $^{\mathbf{a}}$ \\
\hline Parental JFH1-CEtrans or & $\mathrm{n} / \mathrm{a}$ & Open \\
JFH1-CErep & & \\
\hline JFH1-CEtrans-L / JFH1-CErep-L & $\mathrm{C}_{436} \mathrm{G}, \mathrm{A}_{439} \mathrm{U}$ & Open \\
\hline JFH1-CEtrans-R / JFH1-CErep-R & $\mathrm{U}_{496} \mathrm{~A}, \mathrm{G}_{499} \mathrm{C}$ & $\mathrm{Closed}$ \\
\hline JFH1-CEtrans-L/R / JFH1-CErep-L/R & $\mathrm{C}_{436} \mathrm{G}, \mathrm{A}_{439} \mathrm{U}, \mathrm{U}_{496} \mathrm{~A}, \mathrm{G}_{499} \mathrm{C}$ & Open \\
\hline JFH1-CEtrans-S1 / JFH1-CErep-S1 & $\mathrm{U}_{25} \mathrm{~A}, \mathrm{G}_{28} \mathrm{C}$ & Open \\
\hline JFH1-CEtrans-S1/L / JFH1-CErep-S1/L & $\mathrm{U}_{25} \mathrm{~A}, \mathrm{G}_{28} \mathrm{C}, \mathrm{C}_{436} \mathrm{G}, \mathrm{A}_{439} \mathrm{U}$ & $\mathrm{Closed}$ \\
\hline JFH1-CEtrans-S1/R / JFH1-CErep-S1/R & $\mathrm{U}_{25} \mathrm{~A}, \mathrm{G}_{28} \mathrm{C}, \mathrm{U}_{496} \mathrm{~A}, \mathrm{G}_{499} \mathrm{C}$ & Open \\
\hline JFH1-CEtrans-S1/L/R / JFH1-CErep-S1/L/R & $\mathrm{U}_{25} \mathrm{~A}, \mathrm{G}_{28} \mathrm{C}, \mathrm{C}_{436} \mathrm{G}, \mathrm{A}_{439} \mathrm{U}$, & $\mathrm{Closed}$ \\
\hline
\end{tabular}

$3 \mathrm{a}$ in the presence of $\mathrm{miR} 122$

4 